\title{
AN EXAMINATION OF SELECTED DATACOM OPTIONS FOR THE NEAR- TERM IMPLEMENTATION OF TRAJECTORY BASED OPERATIONS
}

\author{
Walter W. Johnson, NASA Ames Research Center, Moffett Field, California \\ Joel Lachter, Vernol Battiste, Veranika Lim, Summer L. Brandt, Robert W. Koteskey, Arik-Quang V. \\ Dao, Sarah V. Ligda, Shu-Chieh Wu, San Jose State University, Moffett Field, California
}

\begin{abstract}
A primary feature of the Next Generation Air Transportation System (NextGen) is trajectory based operations (TBO). Under TBO, aircraft flight plans are known to computer systems on the ground that aid in scheduling and separation. The Future Air Navigation System (FANS) was developed to support TBO, but relatively few aircraft in the US are FANSequipped. Thus, any near-term implementation must provide TBO procedures for non-FANS aircraft. Previous research has explored controller clearances, but any implementation must also provide procedures for aircraft requests. The work presented here aims to surface issues surrounding TBO communication procedures for non-FANS aircraft and for aircraft requesting deviations around weather. Three types of communication were explored: Voice, FANS, and ACARS,(Aircraft Communications Addressing and Reporting System). ACARS and FANS are datacom systems that differ in that FANS allows uplinked flight plans to be loaded into the Flight Management System (FMS), while ACARS delivers flight plans as text that must be entered manually via the Control Display Unit (CDU). Sixteen pilots (eight two-person flight decks) and four controllers participated in 32 20-minute scenarios that required the flight decks to navigate through convective weather as they approached their top of descents (TODs). Findings: The rate of non-conformance was higher than anticipated, with aircraft off path more than $20 \%$ of the time. Controllers did not differentiate between the ACARS and FANS datacom, and were mixed in their preference for Voice vs. datacom (ACARS and FANS). Pilots uniformly preferred Voice to datacom, particularly ACARS. Much of their dislike appears to result from the slow response times in the datacom conditions. As a result, participants frequently resorted to voice communication. These results imply that, before implementing TBO in environments where pilots make weather deviation requests, further research is needed to develop communication procedures that integrate voice and datacom.
\end{abstract}

\section{Introduction}

The US federal government has developed a plan for the Next Generation Air Transportation System (NextGen) that seeks to expand the capacity of the national airspace system (NAS) [1] by transforming air traffic control from clearance-based to trajectory-based operations (TBO). Under TBO, all aircraft fly negotiated 3D/4D trajectories from gate to gate. The advantage of $\mathrm{TBO}$ is that it makes it possible to more accurately predict future aircraft position, and in turn allows ground-based trajectory automation to monitor and ensure safe and efficient separation and scheduling. However, these benefits accrue only so long as aircraft stay on trajectories.

Controller-pilot communication is at the heart of keeping aircraft on trajectories. In current operations when a flight has to divert for weather or to avoid a traffic conflict, controllers typically issue vectors that take aircraft off of their flight plans. In TBO however, the flight plan itself is changed. In order to do this, information that maintains a complete connected trajectory needs to be exchanged between air and ground. The Future Air Navigation System (FANS) has been developed for this purpose but airlines have been slow to equip their aircraft with the moderately expensive FANS equipment since TBO is not presently used in the NAS; while the FAA has not implemented FANS procedures for the NAS (in part) because few aircraft are equipped to use them.

The goal of the current research was to surface issues that may arise in a near-term implementation of TBO. In the long term, most aircraft may have a system such as FANS for air-ground trajectory exchanges, coupled with automation that can specify conflict-free [2] and weather-free paths [3]. However, any near-term implementation of TBO must include procedures for communicating trajectories to aircraft that are not FANS equipped and procedures for pilots to request trajectory deviations, particularly for weather avoidance. We 
developed such procedures for aircraft with three datacom communication equipage levels: Integrated datacom, Non-integrated datacom and Voice only. Integrated Datacom aircraft communicated using a simulation of the FANS-1/A interface currently available on some aircraft. These "FANS" aircraft were able to directly send downlinks and receive uplinks using the Flight Management System (FMS). Non-integrated datacom aircraft communicated using a simulated ACARS Control Display Unit (CDU). These "ACARS" aircraft had text-only datacom with no ability to automatically load uplinks directly to the FMS. Thus, flight plans delivered by ACARS had to be manually entered into the FMS via the CDU. The "Voice" only aircraft had no datacom.

Currently, the ground infrastructure supporting FANS and ACARS is quite different. FANS is used for direct communication with Air Traffic Control (ATC), which uses it to uplink routes. It is almost exclusively used in oceanic operations. ACARS is used for communications between an aircraft and flight dispatch about strategic planning issues such as delays and weather re-routing. Despite the differences between them, it may be possible to capitalize upon the ACARS infrastructure to provide a limited "FANS-like" datacom with ATC for nonFANS equipped flight decks. This may be quite valuable since, while we believe there will be little change to fleet equipage in the near term, we believe the FAA is in a position to act relatively quickly to update its own facilities, in particular to provide an integration of ACARS datacom with ATC tools. Based upon this, we developed TBO procedures that assumed such an integration with a NextGen ATC station. A goal of this ACARS-ATC integration was to make the procedures look and feel as similar to that of FANS as possible, simplifying the ATC task.

\section{Prior Research}

Efficient and accurate controller-pilot communication is critical to safe flight operations. Not surprisingly, errors do occur and are often associated with long ATC messages sent by the controllers with the intention to reduce their own workload [4]. FANS controller-pilot data link communication (CPDLC) amends traditional radio voice communication with a written message capability. Therefore, FANS is better equipped to handle clearances, advisories or warning messages and provide a potential medium to directly interface with the Flight Management System (FMS). In fact, analysis of recorded communications between en route controllers and pilots showed increased controller efficiency and reduced workload in mixedmedia environments incorporating both voice and datacom communications than in voice-only environments [5]. Such findings lend empirical support to the notion that CPDLC may be a superior method of communication.

Other research, however, has found that voice communication remains essential and the combination of voice and datacom communications outperforms individual modes [6]. Specifically, Smith, Lee, Prevot et al. [7] found that voice and CPDLC each has its own set of unique advantages. They examined route negotiations between controllers and pilots using CPDLC or voice modes. They found that requests sent via CPDLC by pilots can provide clearer intent to the controllers than voice requests. On the other hand, they found that voice requests appear more salient to the controllers than do visual (text) requests, especially when they are busy.

On the flight deck, mixed media environments (voice and datacom) have yielded mixed results: under time pressure mixing voice and datacom messages did not help capitalize on the advantages of either medium [8,9]. Closely spaced voice and datacom messages increased the number of requests for clarification for voice messages and the need to review the $\log$ for datacom messages. Others found that datacom communications also changed the nature of crew communication [10]. While the availability of datacom reduced the amount of ATC voice communications, within-crew communication regarding datacom messages increased, resulting in an overall increase in communication time.

The utility of having advanced communication modes cannot be fully exploited without proper procedures that take into account their strengths and weaknesses. There has been very limited research on designing procedures for CPDLC especially in the context of TBO. Mueller [11] examined the possibility of mapping 4D strategic trajectory clearances that included metering, direct routing, and user-preferred routes to datacom messages that can be sent using FANS. Controllers used a trial planner to design strategic trajectory clearances that, upon approval, were sent as FANS messages to the 
aircraft. These clearances were then loaded and executed by the flight deck crew without intervention. Mueller found that the FANS-1/A, infrastructure and the prototype ground automation system appear to be able to satisfactorily implement trajectory-based clearances from controllers.

In a follow-up study, Mueller and Lozito examined flight deck procedures for trajectory-based clearance negotiations [12]. They compared procedures for handling uplinked strategic trajectory clearances that varied the flight deck responsibility between the pilot flying and the pilot monitoring (sharing versus non-sharing) in one simulation study and whether to print datacom clearances in another. All procedures evaluated were rated to be generally suitable.

\section{Objectives}

The current research addresses two major challenges to near term implementation of TBO. First, previous research on designing procedures for strategic trajectory-based clearance negotiations has assumed FANS-1/A flight deck communication equipage [11,12]; however, the current fleet is simply not equipped for broad adoption of FANS procedures. Therefore, the current research explores possible implementations of $\mathrm{TBO}$ using voice procedures or, alternatively, making use of the ACARS datacom found on most transport category aircraft. Second, previous research on flight deck TBO procedures has focused on flight deck review and execution of clearances. While these issues are part of the current research, a primary focus in this study is on flight deck generated requests. Specifically, the current research looks at how aircraft could formulate requests using FANS, ACARS or voice in a TBO environment.

To test our procedures for formulating requests, a human-in-the-loop simulation was conducted. In this simulation pilots and controllers engaged in route negotiation in en route environments with three types of flight deck communication equipage: FANS, ACARS, and voice only. The study uses weather as a way to drive communication. En route weather poses a special problem because alterations of flight plans must be done during flights. An additional complicating factor is that the air and ground have access to different information. In these cases, flight decks bear the responsibility for avoiding weather while ATC bears the responsibility for preventing conflicts. As a result, negotiation is necessary to achieve solutions that satisfy both air and ground. Further, when gaps in a storm front are sufficiently narrow, requests from the air to fly through them may be rejected due to congestion, and clearances from the ground to avoid these congested pathways may be rejected by the air because they are blocked by, or come too close to, weather.

The current research sought to uncover potential problems for near-term implementation of TBO by 'stress testing' the system. As such, we attempted to keep all planes on trajectories and to minimize radio use on datacom equipped aircraft. Therefore, except for safety of flight issues, we steered our air traffic controllers away from solutions that put aircraft on open trajectories (e.g., vectors or off-trajectory climbs and descents), and we told our pilots and controllers not to use radio for aircraft assigned to the FANS and ACARS conditions (again except for safety of flight). Relaxing these constraints may have improved system performance, and operator acceptance, but would not have served our goals.

\section{Concept of Operation}

In this simulation, the controller was responsible for managing approximately 12 aircraft in a high altitude sector in Kansas City Center's airspace (ZKC 90). The sector is on the center boundary adjacent to Indianapolis Center. The primary sector traffic in our simulation was the normal en route traffic flows in this sector along with UPS arrivals into UPS's HUB at Louisville International Airport. Controllers, as in today's operation, were responsible for aircraft separation and traffic management, while pilots were responsible for weather avoidance. Controllers were also responsible for maintaining trajectory-based operations if possible. To accomplish this task, they were instructed to minimize vectoring of aircraft by creating flight plan modifications using the trial planner [13] and delivering the modified trajectories via voice or datacom based on aircraft equipage type. In designing the datacom protocols, one of the goals of the study was to reduce the complexity and workload of managing three differently equipped aircraft types. Thus, from the controller perspective, sending and receiving information from a FANS and ACARS aircraft required the same actions on the controller Display System Replacement (DSR) station. However, the controllers were briefed on the 
flight deck procedures for loading and executing datacom clearances.

There was an important difference between information available to controllers and pilots when handling weather deviation requests sent by either datacom or voice. The controllers constructed trial plans relative to traffic and weather on the DSR display, while pilot requests were based on flight deck weather radar. The NextRad weather (on the controller's display) updated at 5-minute intervals. A bug in the simulation prevented updating of the (ground referenced) location of the weather on the flight deck (see Scenarios below).

Initial procedures for the flight deck were developed by the authors, one of whom is a current airline pilot, and another who is a former air traffic controller. They were then vetted by two other retired controllers, and two pilots. The goal of all procedures was to keep an updated flight plan in a ground based "host" computer, and to make it possible for the aircraft to closely adhere to those flight plans.

An assumption was that, in the near-term, the ground will have an advanced interface for displaying and modifying aircraft trajectories such as that proposed by Prevot [13]. It was also assumed that all flight decks have the ability to fly a track (rather than heading). In current day operations, it is typical for controllers to issue clearances for headings and monitor the aircraft to see that the heading achieves the desired track. In accordance with the general goal of NextGen to reduce controller workload, we felt that such monitoring could just as easily be performed by almost all modern commercial flight decks.

Verbal communication of arbitrary waypoints (lat-longs) were judged to be potentially too error prone. Similarly, extended clearances containing multiple waypoints were judged to be a potential source of error. As a result, our procedures for managing such aircraft called for controllers to develop multiple waypoint clearances using a trial planner that calculated the initial track to the next waypoint. Then the controller monitors the aircraft's progress, issuing multiple track or simple "direct to" clearances, as needed to keep them on route.

For planes that are FANS equipped, the crew can load clearances directly into the FMS. Thus, it is feasible to create arbitrary clearances and upload them to the aircraft.

For planes that are ACARS equipped, the situation is more complicated. Flight plans can be sent to such aircraft as text messages. This reduces the opportunity for communication error. Thus, it was felt that flight plans with arbitrary waypoints such as those sent to FANS aircraft could be uploaded. However, the crew must then manually load such flight plans into the FMS, a potentially timeconsuming process. To assure that the plane remained on its flight path while the waypoint was being entered into the FMS, procedures were developed that allowed pilots to keep the aircraft on its trajectory using the autopilot until the clearance was entered (see 'Flight Deck Procedures' below).

\section{Method}

\section{Participants}

Sixteen commercial airline pilots with glass cockpit experience (eight per week) and four retired TRACON controllers (two per week) were recruited for this simulation. Participant controllers were recruited based on their involvement in previous, similar simulations and were thus familiar with prototype ATC tools used in this simulation. Pilots were divided into two-person crews with the more experienced chosen as the captain. They remained together for the duration of their participation. The simulation ran from August 16-27, 2010 with individual participants running in one of the two weeks. Due to unexpected events, a first officer from the second week dropped out part way through the simulation and was replaced with a captain from the first week. All pilots and controllers were compensated for their participation.

\section{Equipment}

Four dual-pilot stations and two controller stations were operated by study participants. In direct support of the simulation, researchers and confederates operated a simulation manager station and conducted training and observation of all participants. 


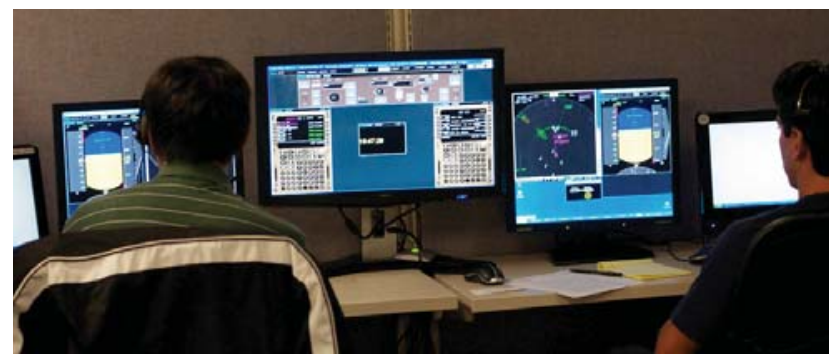

Figure 1. A Dual-Pilot Station

\section{Dual Pilot Stations}

The dual-pilot station consisted of three monitors (see Fig. 1), on which the controls and displays necessary for operating a generic Boeing transport category aircraft were simulated. The paths of participant aircraft were manipulated using the Multi-Aircraft Control System (MACS) [14] autopilot Mode Control Panel (MCP) and dual Control Display Units (CDU's). Flight path and navigation information was presented on dual Cockpit Situation Displays (CSD's) and dual Primary Flight Displays (PFD's). Controls for hand flying the aircraft (e.g., yoke) were not available.

The middle screen of the station was a shared touch screen monitor accessed by both pilots. It hosted the autopilot MCP, the two CDUs, and a text display consisting of a clock and message alerting. The left and right screens of the station each contained a CSD and a PFD for the pilot on that side. Displays on the two side screens were controlled by using a mouse, one for each pilot.

The autopilot MCP, CDUs, PFDs and datacom message display were driven by MACS. The autopilot MCP and PFD's operated as on most Boeing transport category aircraft, while the CDU's operated like a Boeing/Honeywell unit and were used for datacom communications and inputting flight path modifications. The CSD was developed by NASA Ames Flight Deck Display Research Laboratory [15] and presented a 2D display of navigation information, weather radar targets and traffic out to $40 \mathrm{~nm}$ (similar to that displayed by the traffic collision avoidance system, TCAS).

\section{Controller Stations}

For controller stations, MACS was implemented in a controller mode, allowing air traffic controllers to track and manage aircraft in their assigned sector. Controller tools included datacom, conflict alerting and a trial planner for route modifications.
Each participant also had a separate touchscreen computer used to collect workload and flight plan acceptability ratings. For a discussion of this data see Brandt et al. [16].

\section{General Procedures}

Each week began with one day devoted to training, three days scheduled for data collection and a final day for make-up trials and debriefing. Participants were informed that weather and traffic issues would be present which would necessitate negotiation between the flight deck and ATC.

Two simulation worlds were run simultaneously, each containing one participant controller and two participant dual-pilot flight decks. Also present were non-participant aircraft flown by confederate pseudopilots to provide a realistic traffic load of approximately 12 aircraft at any time. Confederate "ghost controllers" managed traffic outside the experimental sector.

\section{Scenarios}

In each world, the dual-pilot flight decks were arrival aircraft headed for Louisville International Airport (SDF), reaching top of descent near the eastern edge of the sector. Participants flew west to east through the sector and negotiated a storm front on the eastern side of the sector.

There were four starting conditions at the beginning of the scenario as defined by the location of the weather and traffic. On the controller displays the weather for each of these four starting conditions evolved in various ways creating 16 total scenarios. Because the weather evolved differently in each case, controllers could not make assumptions about an optimal path through the weather until they watched it develop. A bug prevented movement of the weather on the flight deck. Thus the discrepancy between the location of weather seen on the flight deck and that seen on the ground was somewhat larger than intended. The entire simulation consisted of thirtytwo 20-minute scenarios.

\section{Experimental Design}

The experimental design consisted of two fixed factors (Aircraft Equipage, Airspace Mixture) and three random factors (Scenario, Crew, Controller). The Aircraft Equipage factor reflected the datacom communication capability levels of the individual 
participant aircraft. Three capabilities were modeled: FANS, ACARS and voice. Airspace Mixture was the number of variously equipped aircraft in the sector. There were three conditions: Predominantly Voice ( $80 \%$ voice, $20 \%$ FANS), Predominantly FANS (80\% FANS, $20 \%$ voice) and Predominantly ACARS (60\% ACARS, $20 \%$ voice and $20 \%$ FANS). These three conditions reflected three possible systems of managing the current majority of aircraft that are ACARS. The Predominantly Voice condition imagined that, as today, the ACARS system is not used by ATC and ACARS equipped aircraft are managed in the same manner as unequipped aircraft. The Predominantly FANS condition imagines that these aircraft are upgraded to have data communications integrated with the FMS. Finally, the Predominantly ACARS condition imagines that these aircraft are managed using special procedures. It was expected that the Airspace Mixture factor would affect the controller's workload but have little effect on the crew.

Note that the controller managed many pseudopiloted FANS and voice only equipped aircraft in every condition and many ACARS equipped aircraft in the Predominantly ACARS condition. The pseudopiloted aircraft were designed to react similarly to their participant counterparts. Also note that there were no ACARS aircraft in the Predominantly Voice, or Predominantly FANS, Airspace Mixture conditions.

Each aircrew experienced each scenario twice, each time flying a different aircraft. Each aircraft crew performed four times in each of the six FANS and Voice equipage conditions (two times with each controller for that week) and eight times in the ACARS condition (four times with each controller for that week). Each scenario/experimental aircraft combination was used eight times (once per flight crew) once in each of the six FANS and Voice equipage conditions and twice in the ACARS equipage condition.

\section{Initial Maneuver Points}

Procedures were initially developed in which proposed flight plan amendments developed by the ATC contained an initial maneuver point (IMP) two minutes ahead of the aircraft. This was done in order to allow time to implement, negotiate, and possibly reject the proposal before any flight path maneuver began. The IMP was automatically inserted by the ATC's graphical trial planner. On the other hand, requests developed on the flight deck would always be "off-the-nose" since the flight deck only had the FMS's graphical trial planner, which does not generate IMPs. The intention was for the ATC to echo back acceptable Voice and ACARS flight deck requests with an IMP included. For acceptable FANS flight deck requests, the ATC would simply accept them without inserting an IMP since that is how FANS is presently designed to work. However, during training and initial runs, it became apparent that, for Voice aircraft, communicating this IMP, which was always a lat-long coordinate, increased controller workload disproportionally to any benefits in fidelity to the flight path. As a result, the procedures were modified so that, for Voice aircraft, controllers deleted the automatically generated IMP. This resulted in ATC clearances that were off the nose and therefore required immediate flight deck implementation. If there was a significant delay in this implementation (revealed by the ATC display showing the aircraft to be off path) controllers had to amend the flight plan in the host to correspond to the path actually being flown.

\section{Flight Deck Procedures}

\section{Voice Only Aircraft}

Procedures on the flight deck for Voice aircraft were similar to those followed today, with the exception that trajectory-based requests and clearances were required. Thus, although pilots followed ATC clearances as today, these clearances often took the form of adding a waypoint (e.g., "UPS123, direct HILLS then PRINC remainder of the route unchanged") rather than the unconnected vectors given today. Pilots were to enter this new route into their FMS. Similarly, when making a request, flight decks could request a deviation for weather, but were encouraged to select a named waypoint and request a deviation direct to that waypoint and then a point at which to return to the original route. In this way, the trajectory was preserved in the host during the deviation.

\section{FANS Equipped Aircraft}

When an uplinked clearance was received on a FANS equipped aircraft, a message appeared on the alerting display on the center monitor. The pilot-notflying was then to navigate to the ATC Messages page on the CDU and load the clearance into the 
FMS. If the clearance appeared acceptable (e.g., was clear of weather), the pilot would respond by sending an accept message via the CDU and execute the flight plan. Otherwise, the pilot would reject the clearance and follow up with a revised request to ATC via datacom or voice.

For requests, pilots developed a modified flight plan in the CDU using standard tools. This route was then downlinked to ATC using the ATC message page on the CDU. Pilots then had to monitor the status of this message to see whether it was accepted or rejected. Accepted requests could be executed. Rejected requests were typically followed up with an amended clearance by ATC.

\section{ACARS Equipped Aircraft}

Clearances uplinked to ACARS aircraft were first translated into a free text format (by automation) that then appeared on the ACARS menu in the CDU (see example, Fig. 2). As with FANS messages, these were announced on the message alerting display. The pilot-not-flying would then navigate to the ACARS messages page on the CDU. The clearance was then read and confirmed with the flying pilot before entered into the FMS on the other CDU.

\section{AT: N3907W08710 PROCEED DIRECT N3945W08635 PRINC REST OF ROUTE UNCHANGED \\ FMS CONTINGENCY: AT TIME 02:05:15Z \\ FLY 055 TRACK. WHEN ABLE DIRECT N3945W08635 PRINC REST OF ROUTE UNCHANGED}

\section{Figure 2. Example ACARS Clearance}

Clearances were presented in two parts. The first part was the clearance itself, which contained the path the aircraft was to fly listed as a series of waypoints (possibly including lat-longs). Second, there was an "FMS contingency" part. This contingency included a procedure to execute if the flight crew was not able to enter the clearance before arriving at the IMP, located $\sim 2$ minutes ahead of the aircraft. The FMS contingency provided the time at which automation had predicted the aircraft would reach the IMP and a track the pilots should fly to the second waypoint. In practice, the pilots usually entered the second waypoint into the CDU and waited for the assigned time to execute the maneuver.
ACARS aircraft accepted clearances by free texting back WWW for Wilco and UNA for Unable, which were interpreted appropriately by the automation. Requests made by ACARS aircraft were completed through the free text function of the ACARS ATC message page.

\section{Controller Procedures}

Datacom requests were logged and ordered on the controller DSR in a special datacom-status list, based on the order in which they were received. However, the controller had discretion on when each request was handled. When a request arrived, it was also coded in the aircraft's data tag. To handle the request, the controller would select an icon in the data tag. If it was a FANS aircraft, the proposed route was shown, while for an ACARS aircraft, its request in the datacom-status list was highlighted. For voice aircraft, controllers handled the requests immediately, or else they were required to copy/remember the requests and ask the aircraft to standby. The procedure for modifying the host flight plan using the trial planner for all aircraft was generally the same. The controller modified the current path by first selecting the data tag route icon to display the route, creating a new waypoint on the original path, and finally dragging that waypoint so the resultant path was clear of weather and traffic. The path would automatically "snap" to a named fix if one was close to the desired location. The controller would uplink the new trajectory to datacom equipped aircraft or deliver the new clearance via voice.

\section{Voice Only Aircraft}

After receiving a request to deviate for weather, the controller would create an off-the-nose modification to the aircraft's current flight plan using the trial planner. This modification would be clear of the displayed weather and traffic. The controller would then communicate the route via voice to the aircraft (e.g., "UPS123, fly track 360, direct FIPEN for weather deviation, then proceed direct PRINC.") If the new route was clear of weather by a safe margin, pilots were to fly the assigned route. If the new route was unacceptable, the pilots would request a further deviation via voice. Note that, because the request was off the nose (did not include an IMP), such approved requests generally resulted in the aircraft being off its trajectory requiring ATC to adjust the flight plan in the host. 


\section{FANS Equipped Aircraft}

After receiving a request to deviate for weather, the controller would view the proposed path. If the path was conflict free and fit within the traffic management plan, the controller uplinked an approval message. Upon receiving approval, the flight crew would execute the requested change. Because the request was off-the-nose, this change would be off-the-nose. If the controller was unable to approve the requested flight path deviation due to safety and other concerns, UNA (for Unable) was uplinked followed by a modified flight path clear of weather and traffic based on the downlinked request.

\section{ACARS Equipped Aircraft}

Downlinked weather deviation requests from an ACARS aircraft were received as free text messages in the controller's datacom list. These were sent as off-the-nose requests to proceed either to a named fix, or for a specified direction and distance, with a return to fix on the original route. The controller would acknowledge the request from the aircraft and then open the trial planner to create a route for the aircraft reflecting this request that would not be offthe-nose, but instead included an IMP. If the proposed route was acceptable and fit within the traffic management plan, the controller uplinked an approval message. For unacceptable routes, the controller could uplink a reject message or a new route created with the trial planner.

\section{Dependent Variables}

A paper-and-pencil workload questionnaire was administered after each trial to collect workload and acceptability ratings for the entire trial. A postsimulation questionnaire was administered after the final trial. The post-simulation questionnaire asked pilots to rate items on a 5-point Likert scale related to concept acceptability, safety, procedures, and simulation realism. Participants were provided space to add comments. In addition to the ratings variables collected from post-trial and post-sim questionnaires, objective data were collected in the form of performance, frequency of negotiations, and duration of negotiations. Performance was measured in three manners: the miles added to the original trajectory by the path modification (path stretch), the number of flight path amendments, and the percent of time the aircraft was not in conformance with the host trajectory (non-conformance - aircraft were tagged as non-conforming when 1 mile off path, or on a track 15 deg discrepant from the nominal trajectory).

\section{Results}

\section{Trajectory Conformance}

Our initial analyses show little difference between conditions in terms of the trajectories flown. ANOVAs conducted separately for Aircraft Equipage and Airspace Mixture found no significant differences in path stretch to avoid weather, time off path, or number of flight path amendments. The lack of effects on path stretch and amendments is not too surprising since the factors that drive flight path changes (e.g., weather, conflicting traffic, distance to top of descent) are built into the scenario and may overwhelm any influence of communication method. However, the absence of any effect of Aircraft Equipage on non-conformance is somewhat surprising, since it could be expected that the FANS condition should have performed best, and the Voice worst. This was not found, but a surprising overall level of non-conformance was found.

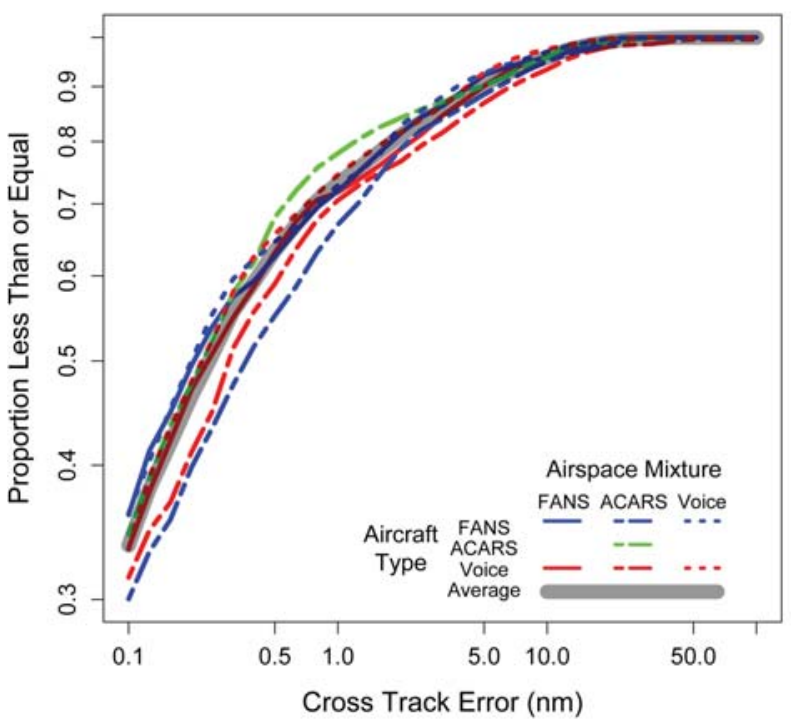

Figure 3. Cross Track Error

Figure 3 shows cumulative distributions for the lateral distance from their trajectory (cross track error) for each combination of Aircraft Equipage and Airspace Mixture as well as the average across all aircraft. In this experiment, controllers were notified when aircraft were more than one nautical mile off 
their trajectory and told to address the problem. Yet aircraft were more than one nautical mile off their trajectories more than $25 \%$ of the time.

\section{Observations}

While we have not systematically categorized all instances of non-conformance, it is clear that much of the non-conformance seen in our data stems from trajectory modification requests made off-the-nose. One reason this caused problems was that we developed procedures that were superficially similar, resulting in some confusion for controllers. For example, with Voice aircraft controllers were told to verify that the request did not result in a conflict by creating an off-the-nose trial plan, entering it into the host, and then approving it verbally. For FANS requests they were told to load the downlinked trajectory and, if conflict free, approve it. For ACARS requests they were told to create a trial plan that had an IMP and uplink that trajectory. Controllers would often combine parts of different procedures, for example, creating an off-the-nose flight plan for an ACARS aircraft. (This would result in aircraft failing to turn as soon as requested while the pilots attempted to enter and vet the new flight path.) Such confusion could probably be reduced by making the procedures more parallel (e.g., never simply approving a request; instead always inserting a maneuver point two minutes ahead of the aircraft), or by adding training that specifically compared and contrasted the differences in these procedures.

Another issue that arises when maneuvers are off-the-nose is that small differences between when the flight plan is loaded into the host on the ground and when it is loaded into the FMS on the aircraft will lead to non-conformance. For instance, if a FANS aircraft downlinks a trajectory request and the ground approves it (simultaneously loading it into the host), the host expects the aircraft to begin the maneuver immediately. If the aircraft is flying eight miles a minute and the pilots delay 8 seconds, they will be out of conformance. Again, this issue might be remedied if a controller uplinked a new flight plan with a specified IMP to FANS aircraft, or if pilots were made more aware of the importance of executing flight plan amendments immediately after they were approved.

While the occurrence of non-conformance could be reduced with modified procedures and training, we are unlikely to be able to prevent aircraft from being out of conformance altogether. Recognizing this, we also gave controllers instructions to adjust the flight path on the DSR when aircraft were late in initiating a maneuver so that it matched the path the aircraft was actually flying. However, it appears that mental workload issues frequently prevented controllers from performing this operation.

\section{Air-Ground Negotiations}

\section{Assumptions and Approach to the Analyses of Negotiations}

Every exchange between pilots and controllers that resulted in a change of flight plan in the aircraft's FMS was operationally defined as a negotiation. Data from visual and verbal recordings, along with digitally recorded datacom was examined for each negotiation to determine the mode (Voice, FANS, or ACARS) used to initiate it, and if Voice was used in combination with datacom. In addition, for each negotiation, the following information was gathered: the time to initial reply or acknowledgment, the initiator (ATC or flight deck), total duration (from first contact to final change of flight plan), and whether the initial proposal was accepted or a revised proposal was necessary. We were primarily interested in negotiations involving weather. Thus, because the modeled storm tops were too high to climb over, the analyses presented here only included negotiations that resulted in lateral flight plan changes.

For most of the comparisons described below we conducted three tests, using controller, scenario, and crew as random factors. This was done to determine if the effects generalized across random variations in these three variables. In most cases where tests were conducted, a significant or marginally significant effect of controller was accompanied by significant effects when using the crew or scenario as a random factor. Therefore, only the F-tests using controller as the random variable are reported.

\section{Number of Negotiations Per Flight}

We categorized the negotiations along two dimensions: Aircraft Equipage (FANS, ACARS or Voice) and Initiator of the negotiation (Flight Deck, or ATC). This parsing showed that, of the 427 negotiations, almost 75\% (304) were initiated from the flight deck. Also, almost all of the ATC-initiated negotiations came later in the scenarios as the ATCs were adjusting the flight paths to ensure timely arrivals to a downstream merge point. 
With respect to the Initiator factor one fact becomes immediately apparent; the number of such negotiations per flight varies by condition. While flight decks initiated requests at roughly equal rates regardless of equipage, controllers give far more clearances to FANS aircraft than ACARS or Voice aircraft (see Figure 4). Three 3x2 ANOVAs were conducted with Aircraft Equipage (FANS, ACARS or Voice) and the initiator of the negotiation (Flight Deck or ATC) as the fixed factors. Significant effects of Aircraft Equipage (F2,6 $=19.33, \mathrm{p}<.01$ by controller) and Initiator $(\mathrm{F} 1,3=69.34, \mathrm{p}<.01$ by controller) were found, while the interaction was marginally significant $(\mathrm{F} 2,6=4.96, \mathrm{p}=.054$ by controller). All effects were significant when using crew and scenario as random variables. The lower proportions of ATC initiated negotiations was expected (since flight decks have the responsibility to request weather avoidance rerouting) but the increased ATC initiated negotiations in the FANS condition was surprising. A follow-up examination of the details of the FANS negotiations did not reveal any obvious explanation.

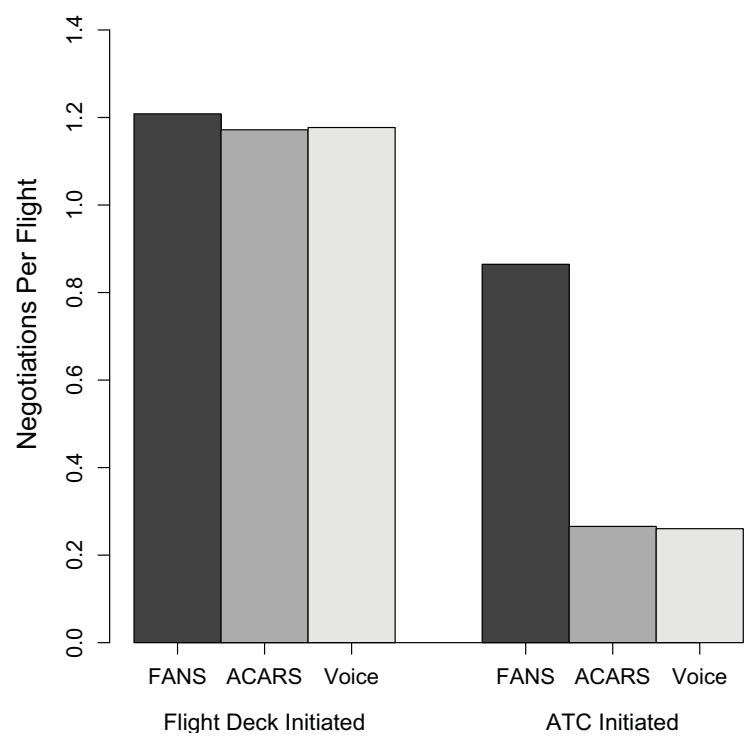

Figure 4. Mean Negotiations Per Flight

\section{Time to Initial Response}

The time taken to respond to a request or clearance is an important step in the negotiating process. Not only does it slow the negotiation down if responses are delayed, the controller or pilot making the request must think of contingencies until they know whether it will be accepted/approved.
Figure 5 shows the time taken to respond as a function of the method used to initiate the negotiation as a "Tukey" boxplot [17]. For each condition, the solid line indicates the median; the box indicates the inter-quartile range (IQR); the whiskers indicate the range excluding "outliers"; and the circles indicate outliers, points more than 1.5 IQR from the box.

There were significant effects of both Aircraft Equipage (F2, $6=10.74, \mathrm{p}<.05$ by controller) and the Initiator $(\mathrm{F} 1,3=10.41, \mathrm{p}<.05$ by controller $)$ as well as their interaction $(\mathrm{F} 2,6=8.17, \mathrm{p}<.05$ by controller). All effects were significant when using scenario and crew as the random variables (since one crew never received an ATC initiated clearance by Voice or ACARS it was removed from the crew analysis here and below).

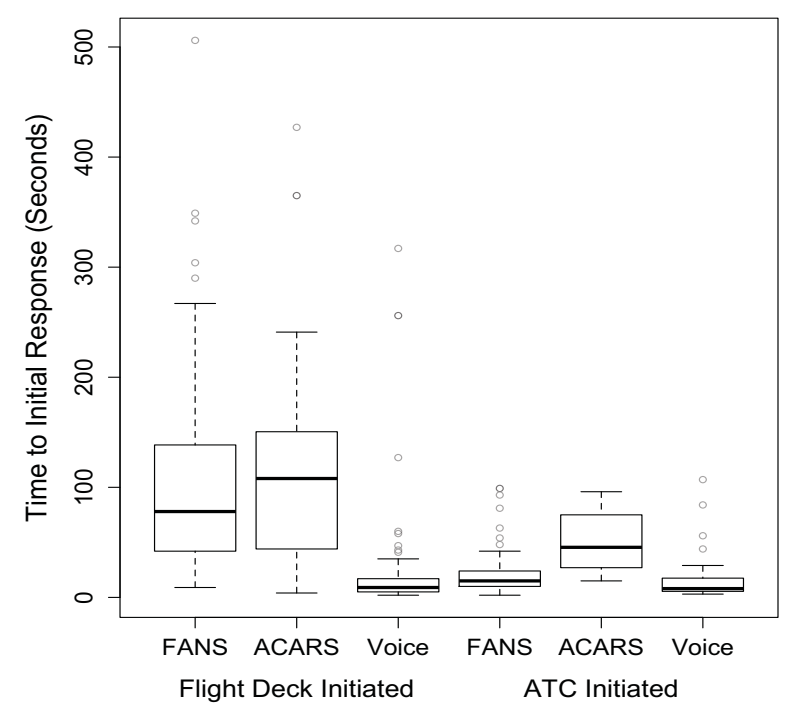

Figure 5. Initial Response Times

Separate analyses showed that controllers took significantly longer to respond to flight deck initiated request sent by FANS than those using Voice $(\mathrm{F} 1,3=$ $20.06, \mathrm{p}<.05$ by controller). A similar trend for ACARS requests was not quite significant by controller $(\mathrm{F} 1,3=9.94, \mathrm{p}=.051$ by controller $)$. Both of these effects were significant by crew and scenario. ACARS and FANS did not differ significantly, with the difference seen in Figure 5 due to a single controller who responded much slower to ACARS than to FANS. However, the remaining controllers all showed small and opposite effects.

While the pattern of flight deck responses to ATC initiated clearances is ordinally consistent with controllers responses to flight deck requests, the 
effects are far smaller and less reliable. Flight deck responses to ACARS clearances were slower than those to FANS, $(\mathrm{F} 1,3=4.70, \mathrm{p}=0.119$, by controller) and slower than those to Voice (F1,3< $6.01, \mathrm{p}=.092$ by controller). However, both of these effects were significant when using scenario and crew as random variables. Responses to FANS were also slower than responses to Voice (but not much), and this was also only marginally significant $(\mathrm{F} 1,3=$ $6.50, \mathrm{p}=.084$, by controller). This effect was significant when analyzed by crew, but not when analyzed by scenario.

That the datacom modes are slower than voice is not surprising for either the flight deck or the ATC. However, the size of the effects for ATC responses is very large, ranging from 1 to 2 minutes - while the activities required to visualize and vet flight deck proposals is probably the greatest for Voice uplinks. Comparatively, flight deck responses to ACARS uplinks were delayed generally less than 1 minute, and this is almost certainly due to 1) the need to key in the request to determine its acceptability and 2) the keystrokes necessary for the flight deck to respond. The few seconds of delay in flight deck responses to FANS (versus Voice) was probably due to the need to physically interact with the CDU.

\section{Choice of Communication Method}

Figure 6 shows the overall proportion of time voice was used (at any time) during negotiations in conditions where datacom was available; and the proportion of time voice was used to initiate negotiations. For example, Figure 6 shows that $40 \%$ of all ATC Initiated negotiations with ACARS equipped aircraft utilized voice at some time during the negotiation, while half of these (about $20 \%$ of the total) began with a voice clearance from ATC.

In addition to the fact that voice was regularly resorted to in the datacom conditions, two main effects can be seen; voice was 1) more likely to be used in negotiations initiated by the flight deck than by negotiations initiated by ATC, and 2) more likely to be used for ACARS than for FANS initiated negotiations. On the other hand, the ATC appears more likely than the flight deck to initiate a negotiation by voice (as compared to just using voice during the negotiation). Due to the small number of voice initiated negotiations (24) and ATC initiated ACARS negotiations using voice at all (17), it was not possible to get reliable estimates of the proportions for the individual crews, controllers, or scenarios. Thus the only significance tests we ran were those comparing overall voice use for FANS and ACARS for the Flight Deck Initiated trials. Here the main effect due to Aircraft Equipage was found to be significant $(\mathrm{F} 1,3=11.74, \mathrm{p}<.05)$. The effect of Aircraft Equipage was significant when using crew and scenario as random variables.

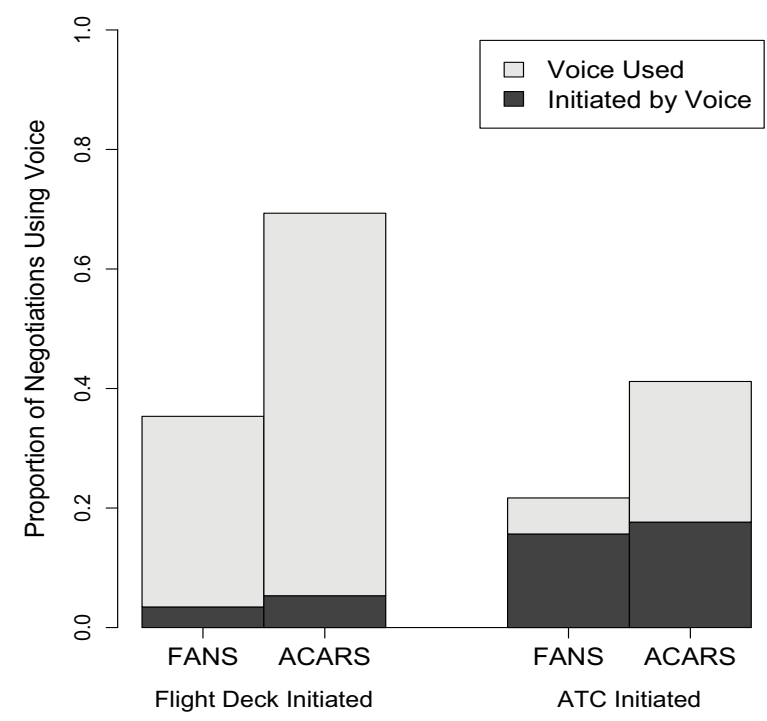

Figure 6. Voice Usage

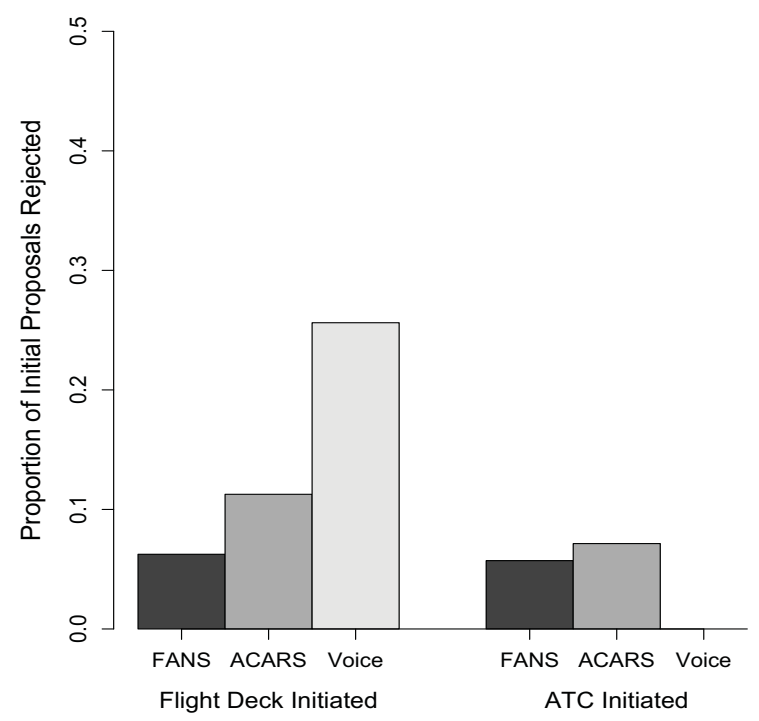

Figure 7. Proposals Rejected

\section{Rejected Proposals}

Both the flight deck and ATC could reject proposed amendments. The proportion of proposals rejected is shown in Figure 7. Similar to the problems encountered with the analysis of voice usage, the numbers of flight deck rejections of ATC initiated 
negotiations are too small, especially for ACARS (14 instances) for reliable estimates of controller, crew or scenario proportions. Therefore only the rejections of the flight deck initiated proposals were analyzed. Here the main effect due to Aircraft Equipage was found to be significant $(\mathrm{F} 2,6=8.13, \mathrm{p}<.05)$.

The relatively high proportion of ATC rejections of flight deck voice proposals stands out. There are several possible explanations for this, including the imprecision of flight deck generated voice proposals and the ease with which the controller is able to suggest alternatives.

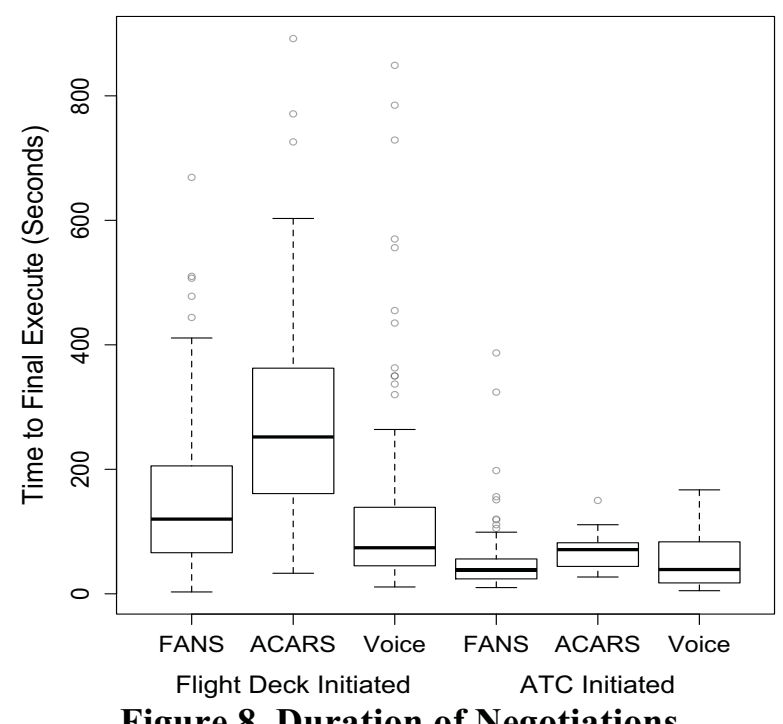

Figure 8. Duration of Negotiations

\section{Duration of Negotiations}

The Figure 8 shows distributions of the duration between the initial contact and the final execution of the newly negotiated flight plan. Clear differences can be seen and were confirmed in two 3 (Aircraft Equipage) x 2 (Initiator) ANOVAs using controller and scenario as random factors. The third random factor, crew, was not used due to the relatively low numbers of ATC initiated negotiations with ACARS and Voice aircraft; this resulted in two crews not contributing data to all conditions. Significant effects for Aircraft Equipage $(\mathrm{F} 2,6=16.26, \mathrm{p}<.01$ by controller), Initiator $(\mathrm{F} 1,3=32.75, \mathrm{p}<.05$ by controller), as well as their interaction ( $F 2,6=9.26$, p $<.05$ by controller), were found. All effects were significant when using scenario as a random variable.

The pattern in the time taken from initial request to execution results from many factors: the method chosen for the interaction (e.g., whether a datacom equipped aircraft chose to contact ATC by voice or datacom), the time taken to respond to the initial request, whether the initially requested trajectory was accepted or further modified and the time taken to implement and execute the new trajectory. One trivial factor is that our procedures for ACARS called for pilots to wait until they reached the initial maneuver point before executing the flight plan change. The pattern however can be seen to be quite similar to that for response to initial request (Figure 5).

\section{Observations}

Perhaps the most interesting result of the negotiations analysis was the prevalent use of voice in the datacom conditions, particularly with ACARS. Some conjectures can be made about the reason for this. First, other analyses showed that the ATC typically took a very long time to respond to flight deck generated datacom requests. Pilots complained about this (see Observations in next section). Therefore, it is likely that the pilots contacted ATC by voice when, in their opinion, the response was not forthcoming. This is especially likely given that response to voice was so immediate. Also, it is possible that voice was used for more complex or difficult requests, or when there was need for more immediate changes to avoid the weather. Finally, in the datacom conditions, the ATC had a greater propensity than pilots to initiate clearances using voice. This suggests that ATC may also have used voice more often in their responses to flight deck generated datacom requests.

Another significant, but puzzling, finding was that ATC initiated requests more often in the FANS condition than in the ACARS or Voice conditions. The reasons for this are not immediately obvious, and need further study.

\section{Subjective Ratings}

\section{Workload Ratings}

At the end of each trial, all participants rated their workload (1 low to 5 high) on four criteria. Pilots were asked to rate Overall and Peak Workload associated with their flight and with weather avoidance, while controllers were asked to rate Overall and Peak Workload associated with maintaining separation and with handling weather avoidance requests.

Four post-trial controller workload ratings were obtained for each level of Airspace Mixture. These 
12 resulting mean workload ratings clustered in a fairly restricted range (from 3.28 to 3.85 ). For these measures, controllers' mean workload was highest in the Predominantly FANS condition, and was lowest for Predominantly Voice, with the exception of Overall workload associated with separation, for which Voice and ACARS were nearly identical. These trends were significant for the "Overall workload associated with separation" (F2,6 =13.905, $\mathrm{p}=.006)$ and "Overall workload associated with weather avoidance requests" (F2,6=5.966, p=.037).

As expected, there was no effect of Airspace Mixture on any pilot workload measure. These varied from 2.10 to 2.77. There were significant effects of Aircraft Equipage; across all workload measures pilots consistently rated their workload highest in the ACARS condition and lowest in the Voice condition. These differences were significant for Overall workload associated with flight, $(\mathrm{F} 2,14=10.310$, $\mathrm{p}=.002)$; Peak workload associated with flight, (F2,14 $=4.811, \mathrm{p}=.026)$; and Overall workload associated with avoiding weather, $(\mathrm{F} 2,14)=5.484, \mathrm{p}=.017)$.

\section{Rating of Procedures}

After the simulation, both pilots and controllers rated how much they agreed with 15 statements (1 low to 5 high) about each of the different communication procedures. For example, pilots were asked to rate their agreement with "I am comfortable with the ACARS/FANS/Voice based trajectory management concept," and "Trajectory operations using ACARS/FANS/Voice are safe." Controllers were asked to rate their level of agreement with "I felt adequately aware of what the pilots of ACARS/FANS/Voice aircraft were doing," and "Trajectory operations using solely ACARS/ FANS/Voice is, in principle, a workable concept."

Controller Ratings: Controllers generally rated the two datacom procedures identically. Two of the four rated ACARS and FANS identical ratings on all 15 statements. That is, they saw no difference in the two procedures on these measures. One controller gave FANS better ratings on three criteria, and the remaining controller gave ACARS a better rating on one criterion. In addition, only one of the four controllers agreed with the statement "I was very aware of whether an aircraft I was handling was integrated datacom communicaiton (FANS-1A) or ACARS." Thus, despite the differences found in the ACARS and FANS negotiation analyses, it appears that our procedures were successful in allowing ACARS-equipped aircraft to be managed similarly to FANS aircraft from the controller's perspective.

While ACARS and FANS appeared very similar to the controllers, Voice, naturally, was quite different. Yet the four controllers differed on whether Voice was preferable to the two datacom conditions (FANS and ACARS). One controller rated the datacom conditions better than Voice on eight of the 15 criteria, while rating Voice better on none. A second controller rated the datacom better on six and Voice better on one. However, a third controller rated Voice better on eight and datacom better on none, and the final controller rated Voice better on one and datacom better on one. Thus, controllers varied in their relative preferences for Voice and datacom.

Pilots Ratings: Unlike the controllers, pilots clearly distinguished between ACARS and FANS. For the twelve statements that allowed us to infer a preference ranking among the three Aircraft Equipage conditions, Voice was always rated best and ACARS always rated worst, with FANS falling in the middle (all statistically significant at the $\mathrm{p}<$ .05 level). This is not surprising since, on the flight deck, pilots were less familiar with ACARS and FANS. In addition ACARS required significantly more work. In ACARS, flight requests had to be manually typed into the ACARS CDU page, while text copies of uplinked flight plans had to be accessed on the CDU ACARS page, and then manually input again into the CDU FMS legs page.

\section{Observations}

It is clear that pilots, as a whole, did not like datacom, especially ACARS. Some insight into this finding can be gleaned from comments gathered during the simulation, responses to open ended questions on the post-simulation questionnaire, and from comments made during the post-simulation debriefing. Pilots had three major concerns about the datacom procedures. The first, and perhaps expected, concern is the time and effort to create and manage messages on the CDU (mentioned by $7 / 16$ for both FANS and ACARS). A second concern was ATC response time (mentioned by 7/16 pilots for FANS and $13 / 16$ for ACARS). In current day operations with voice, the controller responds to messages as they are received. However, in a datacom environment they seem to ascribe priorities in their responses to messages based on traffic management 
needs. For example, if the controller receives a request from an aircraft that will soon be handed off, the controller may respond to it before responding to a previous request by an aircraft in the middle of the sector. Meanwhile, other flight decks wait without feedback that the controller has even received their message. Finally, there were concerns related to message format, such as how the ACARS clearance was formatted, and how the ACARS and FANS messages came up across two pages, thereby requiring additional effort to integrate.

\section{Discussion}

While other studies have shown clear benefits to datacom procedures, our results show a more mixed picture. Controllers showed no clear preference for datacom, and pilots showed quite the opposite, a strong preference for Voice. Similarly, Voice procedures were not worse than datacom procedures in our preliminary examination of performance measures. Why the difference? In our study, most flight path amendments came as a result of pilot requests, while previous work has looked almost entirely at clearances initiated by the controller. Datacom equipage found on current day transport category aircraft makes it difficult to formulate requests and lacks the immediate feedback of traditional voice protocols. The generally slow response time for datacom has been noted in other studies [6,7]. Groce and Boucek [18] specifically note that pilots found datacom unacceptable for weather avoidance clearances because of the time critical nature of such clearances.

It is possible that a communication protocol that combines both datacom and voice could result in more acceptable response times while accruing many of the benefits of datacom (such as reduced transmission error, the ability to transmit more complex clearances, and a reduction in voice traffic). Indeed, pilots and controllers mixed them despite our stated desire that they not do this. For example, a process whereby pilots could initiate requests by voice and receive an acknowledgement by voice, which would then be followed up by datacom exchange, might work better. Several pilots in our study stated that their concerns about datacom would be greatly ameliorated if requests were acknowledged more promptly even if there was a delay in the actual clearance. Such measures might not only increase the acceptability of FANS but also make non-integrated datacom, such as ACARS, acceptable to pilots. This combined approach could also significantly increase options for near-term implementations of TBO. ACARS is just one example of non-integrated datacom, albeit one that is currently available on the majority of transport category aircraft. One could imagine equipping the flight deck with an electronic flight bag (EFB) capable of sending and receiving flight plans. Because of the lower certification standards, EFBs can be a cheaper and more flexible way of upgrading aircraft avionics. The use of EFBs for communicating flight plans might also facilitate deployment of systems with better interfaces for creating requests such as that described in [15].

This study also highlights the difficulties with keeping aircraft on trajectories in an environment where aircraft can make requests. Because no class of aircraft can easily create flight plans that contain IMPs, controllers cannot simply approve requests but must either create a new flight plan and send it back or adjust the flight plan in the host to match what the aircraft is actually flying. Either way adds significantly to the controller workload. One way the controller can ameliorate this problem for himself is to allow planes to go off trajectory. Despite our emphasis in training that aircraft always be on host trajectories, we occasionally observed that a controller appeared unconcerned if one or two flights were not conforming. This may have been because they were allowed to hand off non-conforming aircraft to the next sector, and/or because there were no metering constraints in our study. However, it may also reflect controllers reverting to their standard operational procedures where they consider a trajectory okay so long as they can visually confirm that it contains no conflicts.

While the current study uncovered substantial difficulties with TBO procedures, it should be noted that our scenarios were designed to surface such difficulties should they exist. Pilots might prefer Voice, and controllers might not uniformly prefer datacom on a stormy day when there are many pilot requests. However, benefits for datacom seen in other studies under conditions where it is primarily controllers issuing clearances for separation and interval management are, in all likelihood, real. The current study should not be taken as a reason to question the value of TBO, only as additional caveats on how it should be implemented. 


\section{References}

[1] Joint Planning and Development Office, 2010, Next Generation Transportation System: Concept of Operation V 3.0, Washington D.C, Government Printing Office.

[2] Johnson, W.W., Battiste, V., and Holland, S. (2000) A cockpit display designed to enable limited flight deck separation responsibility. 1999 Transactions of the SAE.

[3] Krozel, J., S. Penny, J. Prete, and J. Mitchell, 2007, Automated route generation for avoiding deterministic weather in transition airspace, vol. 30, J. of Guid., Contr. and Dynamics., pp. 144153.

[4] Morrow, D., A. Lee, and M. Rodvold, 1993, Analysis of problems in routine controller-pilot communication, vol. 3, Int. J. of Av. Psych., pp. 285-302.

[5] Yenson, S. and J. Rakas, 2008, Impacts of a mixed media air traffic control communication environment on aviation efficiency, Athens, Greece, Proc. of the $12^{\text {th }}$ Air Transport Res. Soc. Ann. World Conf.

[6] Kerns, K., 1991, Data-link communication between controllers and pilots: A review and synthesis of the simulation literature, vol. 1, Int. J. of Av. Psych., pp. 181-204.

[7] Smith, N., P. Lee, T. Prevot, J. Mercer, E. Palmer, V. Battiste, V., and W. Johnson, 2004, A Human-in-the-loop Evaluation of Air-Ground Trajectory Negotiation, Chicago, IL, Proc. of the $4^{\text {th }}$ Amer. Inst. of Aero. and Astro. Av. Tech., Integ., and Oper. Conf.,

[8] Lozito, S., L. Martin, M. Dunbar, A. McGann, and S. Verma, 2003, The impact of voice, data link, and mixed air traffic control environments on flight deck procedures, Budapest, Hungary, Proc. of the ATM2003, 5th USA/Eur. R\&D Sem.

[9] McGann, A., D. Morrow, M. Rodvold, and M. Mackintosh,1998, Mixed-media communications on the flight deck: A Comparison of voice, data link, and mixed ATC environments, vol. 8, Int. J. of Av. Psych., pp. 137-156.

[10] Harvey, C., M. Reynolds, A. Pacley, R. Koubek and A. Rehmann, 2002, Effects of the controllerto-pilot data link (datacom) on cres communication, Baltimore, MD, Proc. Of the humfact. and Ergo.Soc. $46^{\text {th }}$ Ann. Meet.

[11] Mueller, E., 2007, Experimental evaluation of an integrated datacom and automation-based strategic trajectory concept, Belfast, Northern Ireland, Proc. of the 7th Amer. Inst. of Aero. and Astro. Av. Tech., Integ. and Oper. Conf.

[12] Mueller, E. and S. Lozito, 2008, Flight deck procedural guidelines for datacom trajectory negotiation, Anchorage, AK, Proc. of the 8th Amer. Inst. of Aero. and Astro. Av. Tech., Integ. and Oper. Conf.

[13] Prevot, T., 2005, On the design of integrated air/ground automation, Waikoloa, Hawaii, IEEE SMC.

[14] Prevot, T., 2002, Exploring the many perspectives of distributed air traffic management: the multi Aircraft Control System MACS, Proc. of the . Int. Conf. on Hum.-Comp. Interaction in Aero., pp. 23-25.

[15] Granada, S., A.-Q. V. Dao, D. Wong, W. W. Johnson, \& V. Battiste, 2005, Development and integration of a human-centered volumetric cockpit situation display for distributed airground operations, Oklahoma City, OK, Proc. of the 12th Int.l Symp. on Av. Psych.

[16] Brandt, S. L., J. Lachter, A.-Q. V. Dao, V. Battiste \& W.W. Johnson, 2011, Flight deck workload and acceptability of verbal and digital communication protocols, in G. Salvendy \& M.J. Smith (Eds.): Human Interface, Part II, HCII 2011, LNCS 6772, pp. 463-472.

[17] Tukey, John W, 1977, Exploratory Data Analysis, Reading, MA, Addison Wesley, 688 p.

[18] Groce, J. L. and G. P. Boucek, 1987, Air transport crew tasking in an ATC data link environment, SAE Tech. Paper Series 871764 Warrendale, PA, Soc of Automotive Engineers.

\section{Acknowledgements}

We would like to thank George Lawton, Dominic Wong, Riva Canton, Tom Quinonez and John Luk for software programming support, and Patrick Cravalho for assistance in recruiting participants. We would especially like to thank Dr. Kim Vu, Dr. Tom Strybel, and the entire California State University, Long Beach, CHAAT Lab staff for their aid in the running and analyses of this study and Doreen Comerford-Roman and Nhut $\mathrm{T}$ Ho for their comments on an earlier draft.

30th Digital Avionics Systems Conference

October 16-20, 2011 